\title{
TWISTING OF PARTIALLY ORIENTED LIQUID CRYSTALS*
}

\author{
BY J. L. ERICKSEN (The Johns Hopkins University)
}

1. Introduction. Liquid crystals consist of relatively rigid molecules, one dimension being large compared to the others. In liquid crystal phases, there is a general tendency for these to align themselves parallel to each other. In isotropic liquid phases, these directions are distributed more or less randomly, at least in fluids at rest. When alignment is nearly perfect, it seems reasonable to attempt to describe these with theories of oriented materials. The molecular interpretation suggests using one director of fixed magnitude, which is the common practice. ${ }^{1}$ Experimentally, there are suggestions of fractional orientation, i.e., of states of orientation intermediate between random and perfect alignment. ${ }^{2}$ In a rather crude way, we might account for this by letting the magnitude of the vector be variable, zero magnitude corresponding to random orientation. Let $\mathbf{n}$ denote the vector. If $\mathbf{n}$ be interpreted as some statistical average of vectors of fixed length associated with individual molecules, its length will never exceed this length. We shall not discuss the rather difficult problem of determining what restrictions this imposes on constitutive equations.

That this mode of describing fractional orientation is crude can be seen by examining molecular theories of, say, solutions involving rigid dumbbell molecules, such as that discussed by Prager [4]. These may be inadequate to describe liquid crystals, but they serve to illustrate the point. In them, calculating the stress involves calculating an average of the type $\left\langle p_{i} p_{i}\right\rangle, p_{i}$ being a relative position vector associated with a molecule. This and averages of the type $\left\langle p_{i} \cdots p_{i}\right\rangle$ can be related through a system of differential equations, in principle infinite in number. In effect, we assume that such averages are simply related to $\left\langle p_{i}\right\rangle$. When alignment is perfect, this is of course correct, but otherwise it involves some error.

Elsewhere [5], I have discussed a one-parameter family of general solutions in the hydrostatic theory of liquid crystals, for the case where $\mathbf{n}$ is constrained to be of fixed magnitude. This involves a twisted orientation pattern of a type commonly observed in liquid crystals of cholesteric type. As is discussed by Brown and Shaw [3, Sec. XG], similar patterns can be produced in liquid crystals of nematic type. Our purpose is to explore what occurs to this pattern when the magnitude of $\mathbf{n}$ is not so constrained.

2. Governing equations. We employ the hydrostatic theory of liquid crystals, as presented by Ericksen [6], assuming incompressibility. This theory involves a stored energy function $W$, interpretable as Helmholz free energy per unit volume, of the form ${ }^{3}$

$$
W=W(\mathbf{n}, \nabla \mathbf{n}) \text {. }
$$

It is subject to the condition that rigidly rotating the material not affect it. Formally,

$$
W\left(\mathbf{R n}, \mathbf{R} \nabla \mathbf{n} \mathbf{R}^{T}\right)=W(\mathbf{n}, \nabla \mathbf{n}),
$$

*Received January 27, 1967.

'See, e.g., Frank [1].

2See, e.g., Peters and Peters [2] or the discussion of the "swarm hypothesis" in Brown and Shaw $[3$, Sec. III $]$.

${ }^{3}$ We use standard direct and Cartesian notations. 
$\mathbf{R}$ being any rotation matrix,

$$
\mathrm{R}^{-1}=\mathrm{R}^{T}, \quad \operatorname{det} \mathrm{R}=1 .
$$

This implies that

$$
A_{i j} \equiv n_{i} \frac{\partial W}{\partial n_{j}}+n_{i, k} \frac{\partial W}{\partial n_{i, k}}+n_{k, i} \frac{\partial W}{\partial n_{k, j}}=A_{i i} .
$$

A common additional assumption is that $\mathbf{n}$ is physically indistinguishable from $-\mathbf{n}$, or

$$
W(-\mathbf{n},-\nabla \mathbf{n})=W(\mathbf{n}, \nabla \mathbf{n}) .
$$

For liquid crystals of nematic type, but not those of cholesteric type, (3) is replaced by

$$
\mathrm{R}^{-1}=\mathrm{R}^{T}, \quad \operatorname{det} \mathrm{R}= \pm 1 .
$$

Generally, applicability of (5) or (6) is inferred from known symmetry properties of the individual molecules in an obvious way.

In the absence of body forces, including some of a generalized nature, ${ }^{4}$ the equations of static equilibrium are, in part,

$$
\partial W / \partial n_{i}-\left(\partial W / \partial n_{i, k}\right)_{, k}=0 .
$$

In terms of $W$, the stress tensor is given by

$$
t_{i j}=-p \delta_{i i}-\tau_{k j} n_{k, i},
$$

where $p$ is an unspecified pressure and

$$
\tau_{k i}=\partial W / \partial n_{k, i} .
$$

The remaining equilibrium equations,

$$
t_{i k, k}=0,
$$

are satisfied if (7) holds and

$$
p=-W+\text { const. }
$$

Let $v$ denote a unit normal to any surface and set

$$
T_{i}=\tau_{i j} \nu_{i} .
$$

We can decompose this vector into a part parallel to $\mathbf{n}$ and a part perpendicular to $\mathbf{n}$,

$$
\begin{aligned}
\mathrm{T} & =\hat{\mathrm{T}}+\hat{\hat{T}} \mathrm{n}, \\
\hat{\hat{T}} n^{2} & =\mathrm{T} \cdot \mathrm{n}, \quad \hat{\mathrm{T}} \cdot \mathrm{n}=0 .
\end{aligned}
$$

The couple stress vector acting on this surface is given by

$$
\mathrm{n} \times \mathrm{T}=\mathrm{n} \times \hat{\mathrm{T}},
$$

the couple stress tensor 1 thus being given by

$$
l_{i i}=\epsilon_{i k p} n_{k} \tau_{p j} .
$$

${ }^{4}$ Some such are associated with body couples resulting from applied electric or magnetic fields. 
Loosely speaking, $\hat{\hat{T}}$ represents a surface "squeezing" action, tending to promote changes in the magnitude of $\mathbf{n}$, while $\hat{\mathbf{T}}$ promotes changes in its direction. There are various observations indicating how one can influence surface orientations. As is discussed by Brown and Shaw [3, Sec. XG], one can produce different surface orientations by putting the liquid crystal in contact with solid single crystals. This forces an orientation depending on the type, cut and orientation of the solid. Techniques for quantitative measurement of the implied couple stress seem not to exist. It is even less clear how one controls perfection of alignment by surface actions, though there are suggestions that some such effect may occur at interfaces.

3. Twisting. Experimentally, the direction of $n$, as a function of position, is inferred from optical observations, frequently using polarized light. In liquid crystals of cholesteric type, it is common to find patterns which, at least locally, are represented by

$$
\mathrm{n}=n(\cos \theta, \sin \theta, 0), \quad \theta=a x_{3}+b,
$$

where $a$ and $b$ are constants. The observed value of $a$ seems to be characteristic of $a$ given material, though it does vary with such factors as temperature and concentration of solutions. As an inverse assumption, we try (15) with $n$ constant, assuming

$$
n>0, \quad a \neq 0 \text {. }
$$

For such a field, it is possible to say much about $W$ and its derivatives, exploiting the invariance of $W$. To a large extent, the reasoning is a repetition of that given by Ericksen [5], so we omit some detail. First, $W$ clearly reduces to a function of $n, \theta$ and $\theta^{\prime}=a$. The effect of applying arbitrary rotations about the $x_{3}$-axis is to change $b$, hence $\theta$ arbitrarily. Since $W$ must be unaffected, as are $n$ and $a$, it must be independent of $\theta$,

A calculation gives

$$
W=f(n, a) \text {. }
$$

$$
\begin{gathered}
n \frac{\partial f}{\partial n}=\frac{\partial W}{\partial n_{i}} n_{i}+\frac{\partial W}{\partial n_{i, 3}} n_{i}^{\prime} \\
\frac{\partial f}{\partial a}=\frac{\partial W}{\partial n_{1}}\left(-x_{3} n_{2}\right)+\frac{\partial W}{\partial n_{2}}\left(x_{3} n_{1}\right)+\frac{\partial W}{\partial n_{1,3}}\left(-a x_{3} n_{1}-n_{2}\right)+\frac{\partial W}{\partial n_{2,3}}\left(-a x_{3} n_{2}+n_{1}\right)
\end{gathered}
$$

a prime denoting differentiation with respect to $x_{3}$. In the latter, the terms explicitly involving $x_{3}$ cancel out, as follows from (4),

so

$$
\partial f / \partial a=n_{1} \partial W / \partial n_{2,3}-n_{2} \partial W / \partial n_{1,3}
$$

Consider

$$
n_{i} \partial W / \partial n_{i, 3} \text {, }
$$

which transforms as a scalar under rotations leaving the $x_{3}$-axis fixed. A repetition of the above argument gives

$$
n_{i} \partial W / \partial n_{i, 3}=g(n, a) .
$$

Now consider a rotation through $180^{\circ}$, with axis parallel to $\mathbf{n}$. The effect should be to reverse the sign of $g$. However, it is easily seen that the arguments of $g$ are unaltered, so

$$
n_{i} \partial W / \partial n_{i, 3}=0 \text {. }
$$


Precisely similar arguments give, for example,

Thus

$$
\begin{aligned}
\partial W / \partial n_{3} & =n_{i} \partial W / \partial n_{3, i}=0, \\
\partial W / \partial n_{3,3} & =h(n, a), \quad n_{i}^{\prime} \partial W / \partial n_{i, 3}=j(n, a) .
\end{aligned}
$$

and

$$
\begin{aligned}
W^{\prime} & =0=\frac{\partial W}{\partial n_{i}} n_{i}^{\prime}+\frac{\partial W}{\partial n_{i, 3}} n_{i}^{\prime \prime} \\
& =\left[\frac{\partial W}{\partial n_{i}}-\left(\frac{\partial W}{\partial n_{i, 3}}\right)_{, 3}\right] n_{i}^{\prime}+\left(\frac{\partial W}{\partial n_{i, 3}} n_{i}^{\prime}\right)^{\prime} \\
& =\left[\frac{\partial W}{\partial n_{i}}-\left(\frac{\partial W}{\partial n_{, j, j}}\right)\right] n_{i}^{\prime},
\end{aligned}
$$

$$
\frac{\partial W}{\partial n_{3}}-\left(\frac{\partial W}{\partial n_{3, j}}\right)_{, j}=\frac{\partial W}{\partial n_{3}}-\left(\frac{\partial W}{\partial n_{3,3}}\right)^{\prime}=0,
$$

whence follows that there exists a scalar $\lambda$ such that

$$
\frac{\partial W}{\partial n_{i}}-\left(\frac{\partial W}{\partial n_{i, j}}\right)_{, i}=\lambda n_{i} .
$$

From (18), (20) and (22), we have

$$
\begin{aligned}
\lambda n^{2} & =\frac{\partial W}{\partial n_{i}} n_{i}-\left(\frac{\partial W^{\prime}}{\partial n_{i, 3}}\right)^{\prime} n_{i}, \\
& =\frac{\partial W}{\partial n_{i}} n_{i}+\frac{\partial W}{\partial n_{i, 3}} n_{i}^{\prime}, \\
& =n \frac{\partial f}{\partial n} .
\end{aligned}
$$

Comparing (7), (22) and (23), we see that (15) is a solution provided the constants $n$ and $a$ are chosen to satisfy the equation

$$
\partial f / \partial n=0 .
$$

With obvious restrictions on the form of $f$, and ignoring the trivial parameter $b$, this will determine one parameter in terms of the other, leaving us with a one-parameter family of solutions.

Symmetry arguments such as are illustrated above, combined with (19) and (24), show that derivatives of $W$ must be of the following form

$$
\begin{aligned}
& \partial W / \partial n_{i}=\left(-a / n^{2}\right)(\partial f / \partial a) n_{i}, \\
& \partial W / \partial n_{3,3}=h(n, a) \\
& \partial W / \partial n_{\alpha, \beta}=h \delta_{\alpha \beta}+k(n, a) n_{\alpha} n_{\beta} ; \quad \alpha, \beta=1,2 \\
& \partial W / \partial n_{1,3}=-\left(1 / n^{2}\right)(\partial f / \partial a) n_{2}, \\
& \partial W / \partial n_{2,3}=\left(1 / n^{2}\right)(\partial f / \partial a) n_{1} \\
& \partial W / \partial n_{3,1}=m(n, a) n_{2} \\
& \partial W / \partial n_{3,2}=-m n_{1} .
\end{aligned}
$$


Further, when (5) applies,

$$
h=k=0 .
$$

This follows from considering the effect of a $180^{\circ}$ rotation about the $x_{3}$-axis, combined with the substitution $\mathbf{n} \rightarrow-\mathbf{n}, \mathbf{n}^{\prime} \rightarrow-\mathbf{n}^{\prime}$. When both (5) and (6) apply, consideration of reflections in the $x_{3}$-direction gives the added simplification

$$
\begin{aligned}
f(n, a) & =f(n,-a), \\
m(n, a) & =-m(n,-a) .
\end{aligned}
$$

That this is as much as can be said in general can be seen by examining special forms of $W$, e.g.

$$
W=F_{1}+F_{2} n_{k, k}+F_{3} n_{i, k} n_{i} n_{k}+F_{4} n_{i, k} n_{k, i}
$$

where the $F$ 's are arbitrary functions of the two invariants

$$
\mathbf{n} \cdot \mathbf{n}, \quad \mathbf{n} \cdot \operatorname{curl} \mathbf{n},
$$

except for obvious restrictions imposed when (5) or (6) applies. With this, it is routine to calculate stress, couple stress, etc. For example, using (13),

$$
n^{2} \hat{T}=\left(h+k n^{2}\right) \mathbf{n} \cdot \boldsymbol{v},
$$

$\hat{T}$ thus being greatest in absolute value when $v$ is parallel to $\mathbf{n}$, as intuition might suggest. Further, from (26), it vanishes for all surfaces when (5) applies. Further, (8), (9) and (25) yield

$$
\left\|t_{i j}\right\|=-p\left\|\begin{array}{lll}
1 & 0 & 0 \\
0 & 1 & 0 \\
0 & 0 & 1
\end{array}\right\|+\left\|\begin{array}{ccc}
0 & 0 & 0 \\
0 & 0 & 0 \\
a h n_{2}, & -a h n_{1}, & -a \partial f / \partial a
\end{array}\right\| .
$$

From (10) and (17), $p$ is constant, so the stresses are uniform. Further, planes $x_{3}=$ const. are subject to no shearing stress, as are planes with $\mathbf{n}$ as normal. When (26) holds, the stress is symmetric, consisting of a uniaxial stress superposed on a hydrostatic pressure. A similar calculation gives

$$
\begin{aligned}
l_{\alpha \beta} & =m\left(n^{2} \delta_{\alpha \beta}-n_{\alpha} n_{\beta}\right), \quad \alpha, \beta=1,2 \\
l_{13} & =-l_{31}=h n_{2}, \\
l_{23} & =-l_{32}=-h n_{1}, \\
l_{33} & =\partial f / \partial a .
\end{aligned}
$$

Various "universal relations" result from eliminating arbitrary functions between these various relations. For example,

$$
a l_{33}=a \partial f / \partial a=t_{11}-t_{33}=t_{22}-t_{33} .
$$

4. Minimum energy. Likely to be of especial interest are solutions which, at least among those here considered, correspond to absolute or relative minimum values of the energy. Presumably, the commonly observed twist of liquid crystals of cholesteric type is singled out by this requirement. One of the competitors is the limiting case

$$
\mathbf{n}=\mathbf{0} \text {, }
$$


which can be shown to be a solution. When this minimizes the energy, the liquid is most likely to be in its isotropic phase, so we ignore this. When (27) holds,

$$
\frac{\partial f}{\partial a}(n, 0)=0, \quad m(n, 0)=0,
$$

which, with (24), indicates that $f$ takes on extremal values with no twist, but a value of $n$ which need not be zero. From (25), (26) and (34) imply that all derivatives of $W$ vanish. For liquid crystals of nematic type, there is some reason to think that such a configuration is of minimum energy, as is discussed by Ericksen [7].

Taking into account (24), necessary conditions for minimum energy are

$$
\begin{gathered}
\frac{\partial f}{\partial a}=0, \\
\frac{\partial^{2} f}{\partial a^{2}}(d a)^{2}+\frac{\partial^{2} f}{\partial n^{2}}(d n)^{2}+2 \frac{\partial^{2} f}{\partial a \partial n} d a a^{2} n \geqq 0, \\
d\left(\frac{\partial f}{\partial n}\right)=0=\frac{\partial^{2} f}{\partial n^{2}} d n+\frac{\partial^{2} f}{\partial a \partial n} d a=0 .
\end{gathered}
$$

Attendant simplifications of the stress and couple stress can be read off from (31) and (32). For example, when (26) holds, the stress reduces to a hydrostatic pressure and the planes $x_{3}=$ const. are free of couple stress. Elimination of $d n$ between (36) and (37), yields the inequality

$$
\frac{\partial^{2} f}{\partial n^{2}}\left[\frac{\partial^{2} f}{\partial n^{2}} \frac{\partial^{2} f}{\partial a^{2}}-\left(\frac{\partial^{2} f}{\partial a \partial n}\right)^{2}\right] \geqq 0 .
$$

For such an extremal twist, it is not necessary that all couple stresses vanish.

Acknowledgment. This work was supported by a grant from the National Science Foundation.

\section{REFERENCES}

[1] F. C. Frank, Discuss. Faraday Soc. 25, 19-28 (1958)

[2] S. Peters and H. Peters, Z. Physik. Chem. (Frankfurt) 3, 103-125 (1955)

[3] Glenn H. Brown and Wilfrid G. Shaw, Chem. Rev. 57, 1049-1157 (1957)

[4] S. Prager, Trans. Soc. Rheol. 1, 53-62 (1957)

[5] J. L. Ericksen, Twisting of liquid crystal, to appear in J. Fluid Mech.

[6] J. L. Ericksen, Arch. Rational Mech. Anal. 9, 371-378 (1962)

[7] J. L. Ericksen, Phys. Fluids 9, 1205-1207 (1966) 